\title{
BMJ Open Exploration of stress management interventions to address psychological stress in stroke survivors: a protocol for a scoping review
}

\author{
Madeleine Hinwood (D) , 1,2 Marina Ilicic, ${ }^{2,3}$ Prajwal Gyawali, ${ }^{2,3,4}$ \\ Murielle Gabriela Kluge, ${ }^{2,3}$ Kirsten Coupland, ${ }^{2,3,5}$ Angela Smith, ${ }^{6}$ \\ Michael Nilsson, ${ }^{2,3,4,7}$ Frederick Rohan Walker $2,3,4,7$
}

To cite: Hinwood M, llicic M, Gyawali P, et al. Exploration of stress management interventions to address psychological stress in stroke survivors: a protocol for a scoping review. BMJ Open 2020;10:e035592. doi:10.1136/ bmjopen-2019-035592

- Prepublication history and additional material for this paper are available online. To view these files, please visit the journal online (http://dx.doi. org/10.1136/bmjopen-2019035592).

MN and FRW are joint senior authors.

Received 07 November 2019 Revised 14 February 2020 Accepted 06 March 2020

D) Check for updates

(c) Author(s) (or their employer(s)) 2020. Re-use permitted under CC BY-NC. No commercial re-use. See rights and permissions. Published by BMJ.

For numbered affiliations see end of article.

\section{Correspondence to} Dr Madeleine Hinwood; Madeleine.Hinwood@newcastle. edu.au

\section{ABSTRACT}

Introduction Several studies have shown that stroke survivors report experiencing high and unremitting levels of stress, which can negatively affect brain repair processes and psychological outcomes and thereby compromise recovery. However, it is presently unclear which interventions have been trialled to manage stress in stroke survivors and whether they translate to clinically relevant outcomes. The aim of this scoping review will be to examine stress management interventions in stroke survivors in order to map the types of interventions trialled, commonly reported stress outcome measures and whether a reduction in stress contributes to reductions in relevant clinical outcomes.

Methods and analysis The methodological framework described in Arksey and 0'Malley will be applied to this review. A draft search strategy was developed in collaboration with an experienced senior health research librarian. A systematic search of Medline, Embase, CINAHL, Cochrane library, Psyclnfo and Clinicaltrials.gov as well as hand searching of reference lists and reviews will identify relevant studies for inclusion. To be eligible for inclusion, studies must report on the outcomes of an intervention targeting stress management and resilience in stroke survivors. Study selection and critical appraisal of selected studies will be carried out independently by two authors, with discrepancies resolved by consensus. Data will be charted using a standard extraction form. Results will be tabulated and narratively summarised to highlight findings relevant to our research questions and to inform recommendations for future research.

Ethics and dissemination This study does not require ethics approval. This scoping review will provide a synthesis of evidence for stress management interventions in stroke survivors. It will identify and clarify the gaps in stress research specific to stroke pathologies and highlight promising interventions for future research. Findings will be relevant to researchers and healthcare workers and will be disseminated via publications in peer-reviewed journals and presented at conferences.

\section{INTRODUCTION}

Stroke affects around 17 million people worldwide per year and is one of the leading causes
Strengths and limitations of this study

- This comprehensive scoping review will collate existing research findings around stress interventions in stroke survivors to inform future research and practice in this important area of stroke care.

- The iterative nature of scoping review data extraction will ensure that all relevant evidence is collected.

- Consideration of the potential feasibility, acceptability and cost-effectiveness of interventions will inform future research, which will assist in scaling up interventions to larger populations.

- The scoping review will include an assessment of study quality to rate existing evidence. This will help to identify potential gaps and areas to validate and address in future work.

- A limitation of this scoping review protocol is the exclusion of grey literature and articles not published in English.

of death and disability worldwide. ${ }^{12}$ The development of medical interventions that reduce the mortality rates of stroke means that a greater number of people survive stroke; however, approximately 25\%-74\% stroke survivors experience ongoing psychological comorbidities including cognitive impairment, anxiety and depression. ${ }^{3-6}$ Identifying risk factors that underlie the development of these disorders is of vital importance to address and improve the recovery phase. Within the general population the development of depression and anxiety is consistently preceded by a stressful life event, which possesses a high level of psychological discomfort or unpleasantness. ${ }^{7}$ The potentially life-changing nature of stroke and the need for ongoing coordination of care services throughout recovery are likely to be significant stressors that contribute to the aetiology of these outcomes for stroke survivors. 
Psychological stress appears to negatively impact the stroke recovery process, with a number of epidemiological studies showing that greater levels of stress poststroke are associated with poorer long-term outcomes. ${ }^{9-13}$ Ostwald $e t a l,{ }^{10}$ in a longitudinal study on the perceived stress of 159 stroke survivors, showed that higher functional impairment in stroke survivors at discharge from inpatient rehabilitation was significantly associated with higher stress levels over a 1-year period. Dos Santos et al 9 showed that perceived stress of stroke survivors after hospital discharge was positively correlated with depressive symptoms, and negatively correlated with functional independence. ${ }^{9}$ Recently, in a cohort study of 182 stroke survivors, Tene $e t a l^{12}$ showed that high salivary cortisol, a stress biomarker, was associated with worse cognitive performance up to 2 years after stroke. In another study from the same cohort, Ben Assayag et $a l^{13}$ showed that higher levels of hair cortisol, thought to represent cumulative stress load over time, predicted worse cognitive outcomes after stroke. Cognitive and psychological domains influenced by stress also contribute to poor motivation and well-being, ${ }^{14}$ which may further negatively impact participation in rehabilitation. Collectively, these studies highlight that stroke survivors with higher levels of stress post-stroke experience worse cognitive, functional and psychological outcomes.

Despite the mounting evidence that stroke survivors experience significant levels of stress connected to worsened cognitive and psychological outcomes, stress is not routinely managed in clinical practice. Stress management techniques have been trialled in the context of cardiovascular disease, however, with varying levels of success. The Enhanced Control of Hypertension and Thrombolysis Stroke Study trial assessed the impact of stress management within a comprehensive cardiac rehabilitation. The inclusion of stress management within a rehabilitation programme resulted in greater reduction in self-reported stress and reduced risk of adverse clinical events (clinical cardiovascular events including myocardial infarction, coronary artery bypass grafts, death, stroke, severe angina and peripheral revascularisation) compared with cardiac rehabilitation alone. ${ }^{15}$ However, several other trials reported no benefit of stress management training over the usual care. A large trial following 2328 postmyocardial infarction patients receiving stress management or usual care showed no group differences in levels of anxiety or depression, or in the incidence of myocardial infarction, cerebrovascular accident, heart failure or revascularisation. ${ }^{16}$ Similarly, Frasure-Smith et $a l^{17}$ showed no differences in anxiety or depression between patients receiving monthly telephone monitoring of psychological distress and patients receiving usual care after myocardial infarction. Additionally, no difference in clinical cardiovascular outcomes was shown. A recent review of mind-body interventions for stress reduction in stroke survivors, including yoga, tai chi and mindfulness-based stress reduction, found that overall five quantitative trials had been published and none reported a significant difference in depression, anxiety or quality-of-life between intervention and control groups. ${ }^{18}$ Overall, data from randomised trials in high-risk populations with cardiovascular disease, and stroke in particular, appears to be limited. The spread of findings suggest that deeper consideration needs to be given to the nature and timing of the interventions, as well as to their intended outcome and the way that the interventions are implemented, resourced and measured. These characteristics will be considered in terms of the success and feasibility of the intervention to provide insight into important differences that may be associated with more beneficial approaches versus those that report no net impact.

Given the prevalence of stress during stroke recovery and its negative impact on the recovery process, the potential for a targeted stress intervention to optimise stroke outcomes is strong. However, due to the variety of interventions that have been trialled, differences in duration of delivery and follow-up, and potentially inconsistent ways of measuring stress (eg, psychometric vs biomarker approaches), at present, there is no clear intervention that can be considered to be more effective than others. Furthermore, it is uncertain whether changes in stress-related outcomes have been translated into clinically relevant changes for stroke survivors. For instance, a corresponding decrease in anxiety and depression improved rehabilitation contacts and improvement in fatigue. Finally, the feasibility of any intervention must be considered; methods of stress intervention may be time consuming and difficult to scale up to larger populations, and acceptability of interventions is also important to improve adherence rates. In this scoping review, we therefore aim to synthesise existing results and approaches around stress interventions in stroke survivors to guide the development and implementation of stress management in future studies and in practice.

\section{Aims and objectives}

The broad aim of this scoping review is to map the available published evidence around stress interventions that have been trialled in stroke survivors. This will provide a comprehensive understanding of current evidence that will be used to clarify the gaps in knowledge and to guide the direction of future research. This protocol is designed to establish a methodology for conducting a scoping review, the aims of which comprise:

- To map the range of interventions trialled addressing stress management in stroke survivors and to identify which interventions show potential for further research.

- To identify potential findings which may help inform guidelines.

- To identify the average duration of follow-up of stress management studies.

- To map the range of outcome measures used for stress.

- To identify whether early intervention for stress translates into a reduction in stress-associated disorders, 
including depression, anxiety and post-traumatic stress disorder.

- To identify barriers and limitations to implementing stress interventions.

- To identify which interventions are feasible and acceptable for stroke survivors.

- To consider the potential cost-effectiveness of stress interventions.

The protocol will be reported using the Preferred Reporting Items for Systematic Review and Meta-Analysis Protocols checklist, ${ }^{19}$ adapted for a scoping review using the Preferred Reporting Items for Systematic Reviews and Meta-Analyses (PRISMA) Extension for Scoping Reviews ${ }^{20}$ (online supplementary appendix 1 ).

\section{METHODS AND ANALYSIS}

We are applying a scoping review methodology as it offers an opportunity to broadly map and summarise existing research findings and identify gaps in existing evidence. We expect this to be an area where the implementation of interventions and measurement of outcomes is likely to be heterogeneous; therefore, a systematic review, which aims to provide a synthesised result for a more specific research question, would not be appropriate. The framework to be used in the scoping review was first outlined by Arksey and $\mathrm{O}^{\prime} \mathrm{Malley}^{21}$ and involves identifying the research question; identifying relevant studies; study selection; charting of the data; and collating, summarising and reporting the results. Methodological approaches by Peters et al, ${ }^{22}$ Levac et $a l^{23}$ and in the Joanna Briggs Institute Reviewers' Manual ${ }^{24}$ will also be considered. Reporting will be conducted according to the PRISMA Extension for Scoping Reviews. ${ }^{20}$ This scoping review was initiated on the 1 March 2019 and is to be completed by January 2020.

\section{Stage 1: identifying the research questions}

A scoping review comprises an iterative process for developing one or more guiding research questions, where each revision is driven by increasing familiarity with the literature. Initially, broad research questions were conceived to ensure that the review would capture the diversity and scope of the literature available. It is our intention to comprehensively examine the literature describing stress interventions that have been trialled in stroke survivors, and as we do so the research questions may be reformulated, and new questions may appear over time. The research questions are outlined in the aims and objectives section of the introduction to this protocol.

\section{Stage 2: identifying relevant studies}

The aim of the scoping review is to comprehensively address broad research questions and will be informed by a systematic search strategy. The initial search strategy was developed in consultation with a senior health research librarian. The strategy drew on the work of the Cochrane Stroke Group to operationalise terms for stroke survivor, stress and intervention study. Publication titles from a preliminary search were reviewed to inform refinement in consultation with the research team. This included the addition of 'resilience' and 'optimism'. The search strategy was developed in Medline and expanded to Embase, PsycInfo, CINAHL and the Cochrane library. Database searches were restricted to human subjects and English language citations only. No date or publication type limits were applied.

We will manually search the reference lists of all included studies as well as relevant systematic reviews. Full details of the initial draft search strategy are included in the supplementary appendix to this manuscript (online supplementary appendix 2). The initial search, which was conducted on 11 March 2019, identified 3820 references, which were imported into Endnote and duplicates eliminated. After deduplication, 2653 references were available for screening.

\section{Stage 3: study selection}

Studies identified in the search will be collated in EndNote X8.2 and exported to Covidence for screening of titles and abstracts, and full-text reviews. All titles and abstracts will be independently reviewed by two authors according to the proposed eligibility criteria (see table 1 ), and conflicts will be resolved in consultation with a third author. When consensus is not reached, that article will be included in the next stage of the review. Following title and abstract screening, full texts of potentially relevant studies will be assessed for eligibility by two authors independently, with any conflicts resolved by consensus among authors. Review authors will communicate regularly during the screening process to discuss study selection, with any alterations recorded. The selection process will be reported using a PRISMA flow diagram. ${ }^{25}$

The proposed inclusion and exclusion criteria are summarised in table 1.

While scoping review methodology does not specify a process for evaluating study quality, we will critically appraise all included papers. This is primarily because the search is limited to intervention studies, and we want to enable both an assessment of the quality of existing evidence and facilitate identification of evidence gaps, including where the quality of existing research does not enable a specific recommendation to be made. We will use The Cochrane Collaboration's tool for assessing risk of bias ${ }^{26}$ to assess the quality of quantitative studies and the Consolidated Criteria for Reporting Qualitative Research checklist ${ }^{27}$ to assess the quality of any qualitative studies that are identified. Study appraisal will be carried out by two independent authors, with any discrepancies found in assessments resolved by consensus among all authors if necessary.

\section{Stage 4: charting the data}

Data charting is the method used for extracting data in scoping reviews. ${ }^{21-24}$ Data charting is a comprehensive assembly of study results and characteristics, which allows 


\begin{tabular}{|c|c|c|}
\hline & Inclusion & Exclusion \\
\hline \multirow[t]{2}{*}{ Population } & Survivors of stroke or transient ischemic attack. & $\begin{array}{l}\text { Non-human subjects (eg, preclinical } \\
\text { studies) }\end{array}$ \\
\hline & \multicolumn{2}{|l|}{ Any age group. } \\
\hline Intervention & Any & Nil exclusion criteria \\
\hline Comparator & Any & Nil exclusion criteria \\
\hline Outcome & Stress resilience & Nil exclusion criteria \\
\hline \multirow[t]{6}{*}{ Publication } & \multirow{6}{*}{$\begin{array}{l}\text { Published intervention studies of any design (quantitative or } \\
\text { qualitative), including randomised controlled trials, quasi- } \\
\text { experimental designs, and pre-test and post-test designs }\end{array}$} & Reviews \\
\hline & & Commentaries/opinion papers \\
\hline & & Letters \\
\hline & & Meta-analyses \\
\hline & & Not published in English \\
\hline & & Unpublished studies (eg, protocols) \\
\hline
\end{tabular}

researchers to capture the span of information within the incorporated studies including context. We will develop a standardised electronic form in Excel to chart the data. For each source, data will be charted by one author and checked by a second author. Any discrepancies will be resolved by consensus among authors. Data extraction will be an iterative process, incorporating an initial trial of data charting and team consultation throughout the process to ensure consistency with review questions and purpose and include new questions that arise during the process. Regular communication between authors will facilitate this process and ensure the reliability of data charting. All significant alterations to the data chart will be recorded.

Preliminary study details and outcomes chosen for charting were guided by The Cochrane Collaboration's Checklist of items to consider in data collection extraction ${ }^{26}$ and the recommendations published in Arksey and O'Malley ${ }^{21}$ and Peters et al. ${ }^{22}{ }^{24}$ Based on preliminary discussions, we developed a number of categories that will guide the extraction and charting of the data from the full-text publications. This is based on providing the data necessary for addressing the main objective of this review, that is, to map the breadth of interventions trialled for stress in stroke populations to date. Presently, these categories include (but are not limited to): bibliographic information; study aims/purpose; research design; number of participants; participant characteristics such as stroke type and sociodemographic; duration of intervention and follow-up; setting; date; country; comorbidities; and all reported outcome measures. Data to be charted on the stress intervention will include frequency, intensity, time and description of intervention and any control or comparison groups; the number of intervention groups; adherence; and satisfaction. Details of outcomes that inform secondary research questions including economic viability, intervention feasibility and acceptability, and intervention safety will be included. If additional data extraction categories emerge during the process, these may be added in consultation with the research team and will be reported along with the findings.

\section{Stage 5: collating, summarising and reporting results}

The purpose of a scoping review is to map research findings and present an overview, rather than systematic synthesis of evidence, which provides results on a narrowly defined question. Therefore, this review is still an iterative work in progress. Using this methodology, we will be able to identify gaps in the research and action areas, which will inform where more in-depth analysis and additional research is required. The results will be summarised and reported to prioritise the findings relevant to the specific research questions. Quantitative data and the results of individual studies' critical appraisal will be tabulated by type of intervention. Any qualitative data will be analysed thematically and reported either narratively or tabulated. Additionally, further narrative description of all results will be provided to aid interpretation of the findings as they relate to the research questions (both those originally defined, as well as new questions that emerge throughout the review process). A narrative approach will also be used to highlight gaps in existing research evidence. The overall results will be used to make recommendations for future research priorities and practice relating to stress management and resilience building techniques for stroke survivors.

The review will be interpreted within the constraints of its limitations. As we are not including grey literature, potentially relevant publications may be missed. Our decision to include only scientific publications was to ensure research quality can be assessed to aid identification of gaps in knowledge and research priorities in future. Any additional limitations identified throughout the scoping review will be acknowledged in the publication of the review.

\section{Statement of patient and public involvement}

There will be no patient or public involvement in this review. 


\section{Ethics and dissemination}

The completed review will be published in a peerreviewed journal, and findings will be further disseminated through presentation at appropriate forums or conferences. Results will also be used to inform the development of future research assessing scalable interventions for stress management in stroke survivors. Only secondary data from published sources will be included in the scoping review; therefore, ethics approval is not required.

\section{DISCUSSION}

Stress is emerging as a significant risk factor for poor stroke recovery outcomes, and the best approach to managing stress within this population must be identified. To the best of our knowledge, this is the first review to map the effectiveness of stress interventions among stroke survivors. This scoping review protocol provides the chosen approach to synthesising a variety of research evidence in a field that is largely disparate. One of the strengths of the proposed review is to identify stress management interventions that could potentially improve the recovery trajectory of stroke survivors. We also anticipate that the findings of this scoping review will identify priorities for future intervention studies and will identify gaps in current stress intervention research, considering the breadth of existing research in the field in relation to efficacy, safety, economics, acceptability and feasibility of interventions for stress and resilience. This review may also have implications beyond stroke care, to consider stress management in other areas of rehabilitation and recovery where stress is likely to play a similarly significant role. The outcomes of this review will be relevant to researchers, clinicians and policymakers both national and internationally.

\section{Author affiliations}

${ }^{1}$ School of Medicine and Public Health, The University of Newcastle, Callaghan, New South Wales, Australia

${ }^{2}$ Hunter Medical Research Institute, New Lambton Heights, New South Wales, Australia

${ }^{3}$ School of Biomedical Sciences and Pharmacy and Priority Research Centre for Stroke and Brain Injury, The University of Newcastle, Callaghan, New South Wales, Australia

${ }^{4}$ NHMRC Centre for Research Excellence in Stroke Rehabilitation and Brain Recovery, Heidelberg, Victoria, Australia

${ }^{5}$ Department of Neurobiology, Care Sciences and Society, Karolinska Institute, Stockholm, Stockholm County, Sweden

${ }^{6}$ HNEHealth Libraries, Hunter New England Local Health District, New Lambton, New South Wales, Australia

${ }^{7}$ Centre for Rehab Innovations, The University of Newcastle, Callaghan, New South Wales, Australia

\section{Twitter Madeleine Hinwood @maddiehinwood}

Contributors MH, FRW and MN conceived and designed the review protocol. MH and $\mathrm{AS}$ designed the draft search strategy. MH, PG, Ml, KC and MGK were involved in conceptualising the research questions, providing input into the search strategy, study selection criteria and plans for data extraction, with FRW and MN providing feedback. All authors contributed to the drafting and editing of the manuscript and approved the final version of the manuscript.
Funding The authors have not declared a specific grant for this research from any funding agency in the public, commercial or not-for-profit sectors.

Competing interests None declared.

Patient and public involvement Patients and/or the public were not involved in the design, or conduct, or reporting, or dissemination plans of this research.

Patient consent for publication Not required.

Provenance and peer review Not commissioned; externally peer reviewed.

Open access This is an open access article distributed in accordance with the Creative Commons Attribution Non Commercial (CC BY-NC 4.0) license, which permits others to distribute, remix, adapt, build upon this work non-commercially, and license their derivative works on different terms, provided the original work is properly cited, appropriate credit is given, any changes made indicated, and the use is non-commercial. See: http://creativecommons.org/licenses/by-nc/4.0/.

ORCID iD

Madeleine Hinwood http://orcid.org/0000-0002-2225-973X

\section{REFERENCES}

1 Béjot Y, Daubail B, Giroud M. Epidemiology of stroke and transient ischemic attacks: current knowledge and perspectives. Rev Neurol 2016;172:59-68.

2 Katan M, Luft A. Global burden of stroke. Semin Neurol 2018;38:208-11.

3 Galligan NG, Hevey D, Coen RF, et al. Clarifying the associations between anxiety, depression and fatigue following stroke. $J$ Health Psychol 2016;21:2863-71.

4 Gillespie DC, Bowen A, Chung CS, et al. Rehabilitation for poststroke cognitive impairment: an overview of recommendations arising from systematic reviews of current evidence. Clin Rehabil 2015;29:120-8.

5 Kapoor A, Lanctôt KL, Bayley M, et al. "Good Outcome" Isn't Good Enough: Cognitive Impairment, Depressive Symptoms, and Social Restrictions in Physically Recovered Stroke Patients. Stroke 2017;48:1688-90.

6 Levine DA, Galecki AT, Langa KM, et al. Trajectory of cognitive decline after incident stroke. JAMA 2015;314:41-51.

7 Miloyan B, Joseph Bienvenu O, Brilot B, et al. Adverse life events and the onset of anxiety disorders. Psychiatry Res 2018;259:488-92.

8 Slavich GM, Stress L. Life stress and health: a review of conceptual issues and recent findings. Teach Psychol 2016;43:346-55.

9 Dos Santos EB, Rodrigues RAP, Fhon JRS, et al. Changes and predictors of psychological stress among elderly stroke survivors 6 months after hospital discharge. Stress Health 2018;34:359-66.

10 Ostwald SK, Bernal MP, Cron SG, et al. Stress experienced by stroke survivors and spousal caregivers during the first year after discharge from inpatient rehabilitation. Top Stroke Rehabil 2009;16:93-104.

11 Ostwald SK, Swank PR, Khan MM. Predictors of functional independence and stress level of stroke survivors at discharge from inpatient rehabilitation. J Cardiovasc Nurs 2008;23:371-7.

12 Tene O, Hallevi H, Korczyn AD, et al. The price of stress: high bedtime salivary cortisol levels are associated with brain atrophy and cognitive decline in stroke survivors. results from the TABASCO prospective cohort study. J Alzheimers Dis 2018;65:1365-75.

13 Ben Assayag E, Tene O, Korczyn AD, et al. High hair cortisol concentrations predict worse cognitive outcome after stroke: results from the TABASCO prospective cohort study. Psychoneuroendocrinology 2017;82:133-9.

14 Praharso NF, Tear MJ, Cruwys T. Stressful life transitions and wellbeing: a comparison of the stress buffering hypothesis and the social identity model of identity change. Psychiatry Res 2017;247:265-75.

15 Blumenthal JA, Sherwood A, Smith PJ, et al. Enhancing cardiac rehabilitation with stress management training: a randomized, clinical efficacy trial. Circulation 2016;133:1341-50.

16 Jones DA, West RR. Psychological rehabilitation after myocardial infarction: multicentre randomised controlled trial. $B M J$ 1996;313:1517-21.

17 Frasure-Smith N, Lespérance F, Prince RH, et al. Randomised trial of home-based psychosocial nursing intervention for patients recovering from myocardial infarction. The Lancet 1997;350:473-9.

18 Love MF, Sharrief A, Chaoul A, et al. Mind-Body interventions, psychological stressors, and quality of life in stroke survivors. Stroke 2019;50:434-40. 
19 Moher D, Shamseer L, Clarke M, et al. Preferred reporting items for systematic review and meta-analysis protocols (PRISMA-P) 2015 statement. Syst Rev 2015;4:1.

20 Tricco AC, Lillie E, Zarin W, et al. PRISMA extension for scoping reviews (PRISMA-ScR): checklist and explanation. Ann Intern Med 2018;169:467-73.

21 Arksey H, O'Malley L. Scoping studies: towards a methodological framework. Int J Soc Res Methodol 2005;8:19-32.

22 Peters MDJ, Godfrey CM, Khalil H, et al. Guidance for conducting systematic scoping reviews. Int J Evid Based Healthc 2015;13:141-6.

23 Levac D, Colquhoun H, O'Brien KK. Scoping studies: advancing the methodology. Implement Sci 2010;5:69.
24 Peters MDJ, Godfrey C, Mclnerney P, et al. Chapter 11: Scoping Reviews. In: Aromataris E, Munn Z, eds. Joanna Briggs Institute Reviewer's Manual. The Joanna Briggs Institute, 2017.

25 Moher D, Liberati A, Tetzlaff J, et al. Preferred reporting items for systematic reviews and meta-analyses: the PRISMA statement. J Clin Epidemiol 2009;62:1006-12.

26 Higgins JP, Green S. Cochrane handbook for systematic reviews of interventions. John Wiley \& Sons, 2011.

27 Tong A, Sainsbury P, Craig J. Consolidated criteria for reporting qualitative research (COREQ): a 32-item checklist for interviews and focus groups. Int J Qual Health Care 2007;19:349-57. 